\title{
Risk Factors for Anxiety in Major Depressive Disorder Patients
}

\author{
$\mathrm{Li}-$ Min Xin ${ }^{1,2}$, Lin Chen ${ }^{2}$, Zhen-Peng $\mathrm{Ji}^{3}$, Suo-Yuan Zhang ${ }^{2}$, Jun Wang ${ }^{2}$, Yan-Hong Liu², Da-Fang Chen ${ }^{3}$, \\ Fu-De Yang ${ }^{2}$, Gang Wang ${ }^{4}$, Yi-Ru Fang ${ }^{5}$, Zheng Lu ${ }^{6}$, Hai-Chen Yang , Jian Hu ${ }^{8}$, Zhi-Yu Chen ${ }^{9}$, Yi Huang ${ }^{10}$, \\ Jing Sun ${ }^{11}$, Xiao-Ping Wang ${ }^{12}$, Hui-Chun $\mathrm{Li}^{13}$, Jin-Bei Zhang ${ }^{14}$, Tian-Mei Si ${ }^{1}$ \\ 'Peking University Sixth Hospital/Institute of Mental Health, National Clinical Research Center for Mental Disorders (Peking University Sixth \\ Hospital), and the Key Laboratory of Mental Health, Ministry of Health (Peking University), Beijing, ${ }^{2}$ Beijing Huilongguan Hospital, Beijing, \\ ${ }^{3}$ School of Public Health, Peking University Health Science Center, Beijing, ${ }^{4}$ Mood Disorders Center, Beijing Anding Hospital, Capital \\ Medical University, Beijing, ${ }^{5}$ Division of Mood Disorders, Shanghai Mental Health Center, Shanghai Jiao Tong University School of Medicine, \\ Shanghai, ${ }^{6}$ Shanghai Tongji Hospital, Tongji University Medical School, Shanghai, ${ }^{7}$ Division of Mood Disorders, Shenzhen Mental Health \\ Centre, Shenzhen, ${ }^{8}$ The First Hospital of Harbin Medical University, Harbin, ${ }^{9}$ Hangzhou Seventh People's Hospital, Hangzhou, ${ }^{10}$ West China \\ Hospital, Sichuan University, Chengdu, ${ }^{11}$ The Affiliated Brain Hospital, Nanjing Medical University, Nanjing, ${ }^{12}$ Mental Health Institute, The \\ Second Xiangya Hospital, Central South University, Changsha, ${ }^{13}$ The Second Affiliated Hospital, College of Medicine, Zhejiang University, \\ Hangzhou, ${ }^{14}$ The Third Affiliated Hospital of Sun Yat-Sen University, Guangzhou, China
}

\begin{abstract}
Objective: To analyze the sociodemographic and clinical factors related to anxiety in patients with major depressive disorder (MDD). Methods: This study involved a secondary analysis of data obtained from the Diagnostic Assessment Service for People with Bipolar Disorders in China (DASP), which was initiated by the Chinese Society of Psychiatry (CSP) and conducted from September 1, 2010 to February 28, 2011. Based on the presence or absence of anxiety-related characteristics, 1,178 MDD patients were classified as suffering from anxious depression ( $n=915)$ or non-anxious depression ( $n=263$ ), respectively.

Results: Compared with the non-anxious group, the anxious-depression group had an older age at onset $(t=-4.39, p<0.001)$, were older ( $t=-4.69, p<0.001$ ), reported more lifetime depressive episodes ( $z=-3.24, p=0.001$ ), were more likely to experience seasonal depressive episodes $\left(\chi^{2}=6.896, p=0.009\right)$ and depressive episodes following stressful life events $\left(\chi^{2}=59.350, p\right.$ $<0.001)$, and were more likely to have a family history of psychiatric disorders $\left(\chi^{2}=6.091, p=0.014\right)$. Their positive and total scores on the Mood Disorder Questionnaire (MDQ) and the 32-item Hypomania Checklist ( $\mathrm{HCL}-32)(p<0.05)$ were also lower. The logistic regression analysis indicated that age (odds ratio $[\mathrm{OR}]=1.03, p<0.001)$, a lower total $\mathrm{MDQ}$ score $(\mathrm{OR}=0.94, p=0.011)$, depressive episodes following stressful life events (OR=3.04, $p<0.001)$, and seasonal depressive episodes $(\mathrm{OR}=1.75, p=0.039)$ were significantly associated with anxious depression.

Conclusion: These findings indicate that older age, fewer subclinical bipolar features, an increased number of depressive episodes following stressful life events, and seasonal depressive episodes may be risk factors for anxiety-related characteristics in patients with MDD.
\end{abstract}

KEY WORDS: Depressive disorder; Anxiety; Risk factors.

\section{INTRODUCTION}

Major depressive disorder (MDD) is a highly prevalent psychological condition associated with high levels of disability, morbidity, and mortality. The Global Burden of Disease study indicated that depression had the third greatest impact on disability-adjusted life-years (DALYs) in Europe and the greatest impact of all physical and men-

\footnotetext{
Received: March 24, 2015 / Revised: April 22, 2015

Accepted: April 27, 2015

Address for correspondence: Tian-Mei Si, MD, PhD

Institute of Mental Health, Peking University, No. 51 Hua Yuan Bei

Road, Hai Dian District, Beijing 100191, China

Tel: +86-10-82801948, Fax: +86-10-62352880

E-mail: si tian-mei@163.com
}

tal diseases in the Americas. ${ }^{1)}$

Comorbid anxiety symptoms or anxiety disorders are common in patients with MDD, and it has been reported that these disorders may share a common underlying pathophysiology. ${ }^{2}$ Anxious depression has been defined as either MDD with high levels of anxiety symptoms (dimensional approach) or as MDD with comorbid/concurrent anxiety disorders (syndromal approach). ${ }^{3)}$ The Sequenced Treatment Alternatives to Relieve Depression (STAR*D) study found that $46.0-53.2 \%$ of participants met criteria for a diagnosis of anxious depression. ${ }^{3,4)}$ In China, Shi et al. ${ }^{5)}$ found that $68.9 \%$ of 508 MDD patients in 15 psychiatric hospitals and clinics suffered from co-

(a) This is an Open-Access article distributed under the terms of the Creative Commons Attribution Non-Commercial License (http://creativecommons.org/licenses/by-nc/4.0) which permits unrestricted non-commercial use, distribution, and reproduction in any medium, provided the original work is properly cited. 
morbid anxiety disorders. These previous studies employed a dimensional assessment approach to define anxious depression, using an anxiety/somatization factor score $\geq 7$ on the 17 -item Hamilton Depression Rating Scale (HAMD-17) ${ }^{6}$ as the cut-off point.

Recent studies have reported that patients with anxious depression exhibited more severe depression, ${ }^{7-9)}$ an increased incidence of suicidal thoughts and behavior, ${ }^{7,10,11)}$ a poorer quality of life, and greater functional impairment than those with non-anxious depression. ${ }^{4,7,12)}$ Studies focused on the treatment of individuals with MDD have demonstrated that comorbid anxiety is associated with a poorer response to antidepressants, ${ }^{8,10,13)}$ a lower remission rate,${ }^{13)}$ an increased likelihood of adverse effects during treatment, ${ }^{13)}$ and an increased rate of recurrence. ${ }^{14)}$ These differences imply that MDD with comorbid anxiety-related characteristics is a more severe form of MDD than MDD alone. Assessment of the anxiety-related characteristics of patients with MDD may be important for the determination of treatment strategies. Few studies have investigated the differences between Chinese patients with MDD with and without anxious characteristics ${ }^{5,713)}$; the extant studies have several limitations, such as the use of a single study site and a small sample. Thus, their results are not generalizable to the Chinese population.

This study aimed to (1) examine the distinctive sociodemographic and clinical characteristics of patients with anxious versus non-anxious depression, and (2) identify the sociodemographic and clinical characteristics associated with the anxiety-related characteristics of patients with MDD patients.

\section{METHODS}

\section{Subjects}

Data were obtained from the Diagnostic Assessment Service for People with Bipolar Disorders in China (DASP), which was initiated by the Chinese Society of Psychiatry (CSP); the study involved patients in 13 major psychiatric hospitals or the psychiatric units of general hospitals, and was conducted from September 1, 2010 to February 28, 2011. Patients aged 16-65 years old with a diagnosis of MDD based on the Diagnostic and Statistical Manual of Mental Disorders 4th edition (DSM-IV) criteria were recruited from the inpatient and outpatient services of 13 major psychiatric hospitals or psychiatric units of general hospitals. All participants were informed of the purpose of the study and provided written consent or verbally agreed to participate; appropriate consent was pro- vided by guardians for participants younger than 18 years of age. The exclusion criteria were as follows: 1 ) a history of or ongoing significant medical or neurological conditions, 2) depressive disorders secondary to a general medical or neurological condition, 3) a previous diagnosis of bipolar disorder, or 4) treatment with electroconvulsive therapy (ECT) within 1 month prior to the study screening. This study protocol was approved by the Ethics Committees of all participating centers.

\section{Instruments}

The diagnostic assessment of MDD was conducted with the Chinese version of the Mini-International Neuropsychiatric Interview (MINI), version 5.0. ${ }^{15,16)}$ Inor outpatients with a diagnosis of MDD who satisfied the study criteria were invited to participate. All research team members were qualified psychiatrists. Patients' basic sociodemographic and clinical data were collected during a clinical interview based on a questionnaire designed for the study; these data were supplemented by a review of medical records, including age at onset, age, educational level, work status, family history of psychiatric disorders, and the presence or absence of frequent depressive episodes, psychotic symptoms, suicidal ideation, and suicide attempts. Frequent depressive episodes were defined as more than four depressive episodes during the previous year. ${ }^{17)}$

MDD patients who experienced one of the anxiety-related characteristics, including excessive worry, anxiety, tension, fearfulness, and fear of loss of control or that bad things will occur were defined as suffering from anxious depression.

The Mood Disorder Questionnaire (MDQ) and the 32-item Hypomania Checklist (HCL-32) are self-administered questionnaires used to identify hypomanic symptoms in patients with MDD ${ }^{18,19)}$ The MDQ consists of 13 items, and the HCL-32 addresses 32 hypomanic symptoms. The questionnaires require yes/no answers, and positive answers are summed to determine the total score. The two tools have been validated in China. Patients with a total MDQ score greater than or equal to 7 were identified as potentially suffering from bipolar disorder (BD) ${ }^{20)}$ whereas patients with a total HCL-32 score greater than or equal to 13 were identified as potentially suffering from $\mathrm{BD}{ }^{20)}$

Prior to the study, all 13 raters were trained to use the MINI with a sample of 20 patients with MDD. The kappa values of each rater were higher than 0.85 . The same raters evaluated the same group of patients throughout the study 
when possible.

\section{Statistical Analysis}

The data were analyzed using SAS 9.1 software (SAS Inc., Cary, NC, USA). Descriptive statistics were used to describe patients' sociodemographic characteristics. Comparisons of the sociodemographic and clinical characteristics or the anxious-depression and non-anxious-depression groups were performed using independent-sample $t$-tests or Mann-Whitney U-tests for continuous data and chi-square tests for categorical data. Multiple logistic regression analysis was used to identify factors associated with the anxious characteristics of MDD patients. Anxiety-related characteristics were treated as dependent variables, whereas sociodemographic and clinical characteristics were treated as independent variables. Statistical significance was set at $p<0.05$ (two-tailed).

\section{RESULTS}

A total of 1,757 patients diagnosed with MDD were invited to participate in the study. Of these patients, 270 (15.4\%) refused or failed to complete the interview, and
309 (17.6\%) patients satisfied the DSM-IV criteria for BD based on the MINI. Ultimately, 1,178 (67.0\%) patients were included in the present analyses. These patients were placed into the anxious-depression $(n=915,77.7 \%)$ or non-anxious-depression ( $\mathrm{n}=263,22.3 \%)$ group according to the presence of anxiety-related characteristics.

Table 1 presents the sociodemographic and clinical characteristics of the anxious-depression and non-anxious-depression groups. Compared with patients with non-anxious depression, those with anxious depression were older $(t=-4.69, p<0.001)$, were older at the onset of the first depressive episode $(t=-4.39, p<0.001)$, had experienced more lifetime depressive episodes $(\mathrm{z}=-3.24$, $p=0.001$ ), were more likely to suffer from depressive episodes following stressful life events $\left(\chi^{2}=59.350, p<0.001\right)$ and from seasonal depressive episodes $\left(\chi^{2}=6.896\right.$, $p=0.009$ ), and were more likely to have a family history of psychiatric disorders ( $\left.\chi^{2}=6.091, p=0.014\right)$; they also had lower positive and total MDQ and HCL-32 scores $(p<0.05)$. The positive and total MDQ and HCL-32 scores in the non-anxious-depression group were higher than those in the anxious group $\left(\chi^{2}=7.283, p=0.007 ; t=2.848, p=0.004\right.$; $\chi^{2}=4.180, p=0.015$; and $t=2.311, p=0.021$, respectively).

Table 1. Comparisons between anxious depression and non-anxious depression groups regarding the basic demographic and clinical variables

\begin{tabular}{|c|c|c|c|c|c|c|}
\hline \multirow{2}{*}{ Item } & \multirow{2}{*}{$\begin{array}{c}\text { Total } \\
(n=1,178)\end{array}$} & \multirow{2}{*}{$\begin{array}{l}\text { Non-anxious } \\
\text { depression } \\
(n=263)\end{array}$} & \multirow{2}{*}{$\begin{array}{c}\text { Anxious } \\
\text { depression } \\
(n=915)\end{array}$} & \multicolumn{3}{|c|}{ Statistics } \\
\hline & & & & $\chi^{2}$ & $d f$ & $p$ value \\
\hline Female & $793(67.3)$ & $173(65.8)$ & $620(67.8)$ & 0.364 & 1 & 0.564 \\
\hline Married & $824(70.0)$ & $174(66.2)$ & $650(71.0)$ & 2.313 & 1 & 0.128 \\
\hline Unemployed & $366(31.1)$ & $92(35.0)$ & $274(29.9)$ & 2.419 & 1 & 0.120 \\
\hline Education (Senior secondary school and below) & $668(56.7)$ & $143(54.4)$ & $525(57.4)$ & 0.751 & 1 & 0.386 \\
\hline $\begin{array}{l}\text { Frequent depressive episodes } \\
\text { ( }>4 \text { in the previous year) }\end{array}$ & $90(7.6)$ & $16(6.1)$ & $74(8.1)$ & 1.162 & 1 & 0.281 \\
\hline $\begin{array}{l}\text { Depressive episodes with increased appetite, } \\
\text { weight gain and time spent sleeping }\end{array}$ & $179(15.2)$ & $36(13.7)$ & $143(15.6)$ & 0.597 & 1 & 0.440 \\
\hline $\begin{array}{l}\text { Depressive episodes with } \\
\text { suicidal ideation or attempts }\end{array}$ & $665(56.4)$ & $136(51.7)$ & $529(57.8)$ & 3.095 & 1 & 0.079 \\
\hline Depressive episodes with psychotic symptoms & $158(13.4)$ & $36(13.7)$ & $122(13.3)$ & 0.022 & 1 & 0.882 \\
\hline Depressive episodes following stressful life events & $578(49.1)$ & $74(28.1)$ & $504(55.1)$ & 59.350 & 1 & $<0.001$ \\
\hline Seasonal depressive episodes & $134(11.4)$ & $18(6.8)$ & $116(12.7)$ & 6.896 & 1 & 0.009 \\
\hline Family history of psychiatric disorders & $203(17.2)$ & $32(12.2)$ & $171(18.7)$ & 6.091 & 1 & 0.014 \\
\hline Antidepressant use & $905(76.8)$ & $197(74.9)$ & $708(77.4)$ & 0.701 & 1 & 0.402 \\
\hline HCL-32 positive & $316(26.8)$ & $86(32.7)$ & $230(25.1)$ & 4.180 & 1 & 0.015 \\
\hline MDQ positive & $141(12.0)$ & $44(16.7)$ & $97(10.6)$ & 7.283 & 1 & 0.007 \\
\hline Age $(y r)$ & $40.5 \pm 12.9$ & $37.3 \pm 12.3$ & $41.5 \pm 12.9$ & $-4.69^{*}$ & 1,170 & $<0.001^{\dagger}$ \\
\hline Age at onset (yr) & $34.6 \pm 12.5$ & $31.8 \pm 11.7$ & $35.5 \pm 12.7$ & $-4.39^{*}$ & 1,170 & $<0.001^{\ddagger}$ \\
\hline Lifetime depressive episodes & $1.9 \pm 2.6$ & $1.6 \pm 2.4$ & $1.9 \pm 2.6$ & $-3.24^{*}$ & - & $0.001^{\S}$ \\
\hline Number of admissions & $0.4 \pm 1.0$ & $0.4 \pm 0.9$ & $0.4 \pm 1.1$ & $-0.40^{*}$ & - & $0.692^{\S}$ \\
\hline Total MDQ score & $7.9 \pm 6.7$ & $3.3 \pm 2.8$ & $2.8 \pm 2.7$ & $2.848^{*}$ & 1,170 & $0.004^{\dagger}$ \\
\hline Total HCL-32 score & $2.9 \pm 2.8$ & $8.7 \pm 6.7$ & $7.6 \pm 6.7$ & $2.311^{*}$ & 1,170 & $0.021^{\dagger}$ \\
\hline
\end{tabular}

Values are presentd as number (\%) or mean \pm standard deviation.

${ }^{*} \mathrm{~T} / \mathrm{Z}$; ${ }^{+}$independent-sample t-tests; ${ }^{2}$ separate variance estimation $t$-test; ${ }^{\S}$ Mann-Whitney U-test.

df, degree of freedom; HCL-32, the 32-item Hypomania Checklist; MDQ, the Mood Disorder Questionnaire. 
Table 2. Multiple logistic regression analysis of the socio-demographic and clinical factors associated with anxious characteristics in major depression disorder patients $(n=1,178)$

\begin{tabular}{lrrrrrr}
\hline & Risk factor & $\beta$ & SE & Wald $\chi^{2}$ & OR & $95 \%$ Cl \\
\hline Age value & 0.02 & 0.01 & 17.79 & 1.03 & $1.01-1.04$ & $<0.001$ \\
Total MDQ score & -0.07 & 0.03 & 6.40 & 0.94 & $0.89-0.99$ & 0.011 \\
Depressive episodes following stressful life events & 1.11 & 0.15 & 51.58 & 3.04 & $2.25-4.20$ & $<0.001$ \\
Seasonal depressive episodes & 0.56 & 0.27 & 4.25 & 1.75 & $1.03-2.99$ & 0.039 \\
\hline
\end{tabular}

$\mathrm{SE}$, standard error; OR, odds ratio; $\mathrm{Cl}$, confidence interval; $\mathrm{MDQ}$, the Mood Disorder Questionnaire.

There were no significant differences between the groups regarding gender, marital status, work status, or education level.

A stepwise logistic regression analysis demonstrated that older age ( $\beta=0.02, \mathrm{OR}=1.03, p<0.001$ ), a lower total MDQ score ( $\beta=-0.07, \mathrm{OR}=0.94, p=0.011)$, depressive episodes following stressful life events $(\beta=1.11$, $\mathrm{OR}=3.04, p<0.001$ ), and an increased number of seasonal depressive episodes $(\beta=0.56, \mathrm{OR}=1.75, p=0.039)$ were associated with comorbid anxiety-related characteristics in patients with MDD (Table 2).

\section{DISCUSSION}

To the best of our knowledge, this study is the first large-scale, multicenter study to examine the distinctive sociodemographic and clinical characteristics of anxious versus non-anxious depressed patients in China. We found that $77.7 \%$ of the MDD patients exhibited anxiety-related characteristics, which is consistent with previous results in China. ${ }^{5)}$ The logistic model identified older age, a lower total MDQ score, depressive episodes following stressful life events, and seasonal depressive episodes as risk factors for comorbid anxiety.

Previous research has demonstrated that patients with anxious depression were more likely to be older than patients with non-anxious depression. ${ }^{3,7,8)}$ In our study, patients with anxious depression were significantly older than those with non-anxious depression (41.5 vs. 37.3 years) according to multivariate analyses. Patients with anxious depression also experienced more lifetime depressive episodes, which is consistent with previous studies showing that such patients were more likely to experience recurrences. ${ }^{11)}$ Magnusson ${ }^{21)}$ reported that approximately $10-20 \%$ of major depressive episodes had seasonal characteristics. The rate of $11.4 \%$ found by our study is consistent with this previous result, and patients with anxious depression were more likely to have seasonal depressive episodes (12.7 vs. 6.8\%). Researchers in Norway who examined 60,995 participants reported that the high- est rate of comorbid depression and anxiety occurred in the spring (April, May) and October. ${ }^{22)}$ Therefore, medical histories should include questions about whether depressive episodes have seasonal characteristics.

Although differences between groups with respect to a family history of psychiatric disorders were evident in the univariate analysis, these differences were not observed in the multivariate analysis. This finding is consistent with previous studies. ${ }^{11,13)}$ However, another study found that the parents of patients with anxious depression had a broader range of psychiatric disorders, including anxiety, depressive disorders and mania, whereas the parents of patients with non-anxious depression were more likely to have only major depression or depression comorbid anxiety disorder. ${ }^{9)}$ Future research should examine family histories of various kinds of psychological disorders.

One retrospective study reported that the diagnosis of $21 \%$ of patients with an initial diagnosis of depressive disorder was changed to other psychiatric diagnoses after 2 years. ${ }^{23)}$ Several studies have indicated that bipolar features could be identified in approximately one-quarter of patients diagnosed with MDD. ${ }^{24-26)}$ The DASP project excluded 309 cases (20.8\%) diagnosed with BP, whereas the 1,178 participants included a subgroup of patients with positive total HCL-32 or MDQ scores. The positive rate in the non-anxious-depression group was higher than that in the anxious-depression group, indicating that patients with non-anxious depression were more likely to have subclinical bipolar features. However, a previous study reported that many patients diagnosed with unipolar depression may have undiagnosed BP-related comorbid anxiety-related conditions. ${ }^{27)}$ Despite the different results, it is important to address the possibility of manic episodes in the course of MDD treatment, regardless of whether there are comorbid anxiety symptoms.

Several research studies have demonstrated that patients with anxious depression were more likely to experience suicidal ideation or attempt suicide than non-anxious depression group. ${ }^{10,11,28)}$ In our study, the rates of suicidal ideation or attempts in the anxious-depression group were 
higher than those in the non-anxious group; however, there was no significant difference between the two groups in this regard, which is consistent with the results reported by Shi et al. ${ }^{5)}$ However, suicidal ideation is closely related to suicidal behavior, ${ }^{29)}$ and the rates of suicidal ideation in the two groups were higher than $50 \%$; thus, suicidal ideation and suicide attempts should be examined in clinical practice.

A previous study indicated that patients with anxious depression were more likely to be female, have less formal education, and be unemployed. ${ }^{3)}$ Other studies have demonstrated that patients with anxious depression were likely to exhibit melancholic features. ${ }^{7,8)}$ However, in our study, no significant differences between the subjects with anxious and non-anxious depression were identified regarding gender, educational level, work status, or comorbid melancholic features. These discrepancies may be explained by cultural differences.

There are several limitations of this study that should be considered when interpreting the results and recommendations. First, no standardized rating scale was used to measure the characteristics of anxiety, but use of such a scale may have provided a more accurate diagnostic picture. Some studies have used the Hamilton Rating Scale for Depression (HAMD) ${ }^{7,8)}$; however, this scale includes only a few items related to anxiety symptoms, and it ignores the subjective feelings of patients. Second, several important variables were not investigated, such as comorbid obsessive-compulsive symptoms, comorbid substance abuse or dependence, and overall functioning. Third, some patients may exhibit manic symptoms and be diagnosed with bipolar disorder in the future. Fourth, our use of a cross-sectional approach allowed investigation only of risk factors related to previous experiences with anxiety, and we cannot confidently use these risk factors to predict such experiences in the future. Fifth, the data related to the age of onset and lifetime depressive episodes were retrospectively collected; thus, memory bias may have affected the main findings.

In conclusion, we demonstrated that patients with anxious depression had an older age of onset, were older, experienced more lifetime depressive episodes, reported fewer subclinical bipolar features, suffered from more seasonal depressive episodes and an increased number of depressive episodes following stressful life events, and were more likely to have a family history of psychiatric disorders. Risk factors, including older age, fewer subclinical bipolar features, depressive episodes following stressful life events, and an increased number of seasonal depressive episodes, were associated with comorbid anxiety-related characteristics accompanying MDD. To improve the treatment and quality of life of these patients, clinicians should attempt to recognize these risk factors early and select treatment strategies accordingly.

\section{Acknowledgments}

This study was supported by the National Key Scientific and Technological Projects (973Program: 2013CB531305), and the National Key Technology R\&D Program (2015BAI13B01). The study was initiated by the Chinese Society of Psychiatry (CSP) with support from AstraZeneca China. AstraZeneca China had no role in the study design or the generation or interpretation of the results. The authors thank all clinicians who helped to organize the study at each study site.

\section{REFERENCES}

1. Ustün TB, Ayuso-Mateos JL, Chatterji S, Mathers C, Murray CJ. Global burden of depressive disorders in the year 2000. Br J Psychiatry 2004;184:386-392.

2. Hettema JM. What is the genetic relationship between anxiety and depression? Am J Med Genet C Semin Med Genet 2008;148C:140-146.

3. Fava M, Alpert JE, Carmin CN, Wisniewski SR, Trivedi $\mathrm{MH}$, Biggs MM, et al. Clinical correlates and symptom patterns of anxious depression among patients with major depressive disorder in STAR*D. Psychol Med 2004;34: 1299-1308.

4. Fava M, Rush AJ, Alpert JE, Balasubramani GK, Wisniewski SR, Carmin CN, et al. Difference in treatment outcome in outpatients with anxious versus nonanxious depression: a STAR*D report. Am J Psychiatry 2008;165: 342-351.

5. Shi SS, Zhang MY, Wu WY, Lu Z, Zhou TX, Zhang HY, et al. Multi-center study of the clinical features in depression comorbidity with anxiety disorders. Shanghai Arch Psychiatry 2009;21:198-202.

6. Hamilton M. A rating scale for depression. J Neurol Neurosurg Psychiatry 1960;23:56-62.

7. Lin $\mathrm{CH}$, Wang FC, Lin SC, Chen CC, Huang CJ. A comparison of inpatients with anxious depression to those with nonanxious depression. Psychiatry Res 2014;220: $855-860$.

8. Wiethoff K, Bauer M, Baghai TC, Möller HJ, Fisher R, Hollinde D, et al. Prevalence and treatment outcome in anxious versus nonanxious depression: results from the German Algorithm Project. J Clin Psychiatry 2010;71: 1047-1054.

9. Goldberg DP, Wittchen HU, Zimmermann P, Pfister H, Beesdo-Baum K. Anxious and non-anxious forms of major depression: familial, personality and symptom characteristics. Psychol Med 2014;44:1223-1234.

10. Goldberg D, Fawcett J. The importance of anxiety in both major depression and bipolar disorder. Depress Anxiety 2012;29:471-478.

11. Seo HJ, Jung YE, Kim TS, Kim JB, Lee MS, Kim JM, et al. Distinctive clinical characteristics and suicidal tendencies 
of patients with anxious depression. J Nerv Ment Dis 2011;199:42-48.

12. Chan HN, Rush AJ, Nierenberg AA, Trivedi M, Wisniewski SR, Balasubramani GK, et al. Correlates and outcomes of depressed out-patients with greater and fewer anxious symptoms: a CO-MED report. Int J Neuropsychopharmacol 2012;15:1387-1399.

13. Wu Z, Chen J, Yuan C, Hong W, Peng D, Zhang C, et al. Difference in remission in a Chinese population with anxious versus nonanxious treatment-resistant depression: a report of OPERATION study. J Affect Disord 2013;150: 834-839.

14. Shi SS, Zhang MY, Wu WY, Lu Z, Zhou TX, Zhang HY, et al. One year outcome of patients with comorbid depression and anxiety disorders. Chin J Psychiatry 2013;46:212-216.

15. Sheehan DV, Lecrubier Y, Sheehan KH, Amorim P, Janavs $\mathrm{J}$, Weiller E, et al. The Mini-International Neuropsychiatric Interview (M.I.N.I.): the development and validation of a structured diagnostic psychiatric interview for DSM-IV and ICD-10. J Clin Psychiatry 1998;59 Suppl 20:22-33;quiz 34-57.

16. Si TM, Shu L, Dang WM, Su YA, Chen JX, Dong WT, et al. Evaluation of the reliability and validity of Chinese version of the Mini International Neuropsychiatric Interview in Patients with Mental Disorders (in Chinese). Chin Ment Health J 2009;23:493-503.

17. Bowden CL. Strategies to reduce misdiagnosis of bipolar depression. Psychiatr Serv 2001;52:51-55.

18. Hirschfeld RM, Williams JB, Spitzer RL, Calabrese JR, Flynn L, Keck PE Jr, et al. Development and validation of a screening instrument for bipolar spectrum disorder: the Mood Disorder Questionnaire. Am J Psychiatry 2000;157: 1873-1875.

19. Angst J, Adolfsson R, Benazzi F, Gamma A, Hantouche E, Meyer TD, et al. The HCL-32: towards a self-assessment tool for hypomanic symptoms in outpatients. J Affect Disord 2005;88:217-233.

20. Yang HC, Yuan CM, Liu TB, Li LJ, Peng HJ, Liao CP, et al. Validity of the 32-item Hypomania Checklist (HCL-32) in a clinical sample with mood disorders in China. BMC Psychiatry 2011;11:84.

21. Magnusson A. An overview of epidemiological studies on seasonal affective disorder. Acta Psychiatr Scand 2000; 101:176-184.

22. Stordal E, Morken G, Mykletun A, Neckelmann D, Dahl AA. Monthly variation in prevalence rates of comorbid depression and anxiety in the general population at 63-65 degrees North: the HUNT study. J Affect Disord 2008; 106:273-278.

23. Kim W, Woo YS, Chae JH, Bahk WM. The diagnostic stability of DSM-IV diagnoses: An examination of major depressive disorder, bipolar I disorder, and schizophrenia in Korean patients. Clin Psychopharmacol Neurosci 2011;9:117-121.

24. Manning JS, Haykal RF, Connor PD, Akiskal HS. On the nature of depressive and anxious states in a family practice setting: the high prevalence of bipolar II and related disorders in a cohort followed longitudinally. Compr Psychiatry 1997;38:102-108.

25. Hantouche EG, Akiskal HS, Lancrenon S, Allilaire JF, Sechter D, Azorin JM, et al. Systematic clinical methodology for validating bipolar-II disorder: data in mid-stream from a French national multi-site study (EPIDEP). J Affect Disord 1998;50:163-173.

26. Benazzi F. Prevalence of bipolar II disorder in outpatient depression: a 203-case study in private practice. J Affect Disord 1997;43:163-166.

27. Bolge SC, Thompson T, Bourne E, Nanry K. Characteristics and symptomatology of patients diagnosed with unipolar depression at risk for undiagnosed bipolar disorder: a bipolar survey. CNS Spectr 2008;13:216-224.

28. Batterham PJ, Christensen H, Calear AL. Anxiety symptoms as precursors of major depression and suicidal ideation. Depress Anxiety 2013;30:908-916.

29. May AM, Klonsky ED, Klein DN. Predicting future suicide attempts among depressed suicide ideators: a 10-year longitudinal study. J Psychiatr Res 2012;46:946-952. 